\title{
Kinetics-Driven Superconducting Gap in Underdoped Cuprate Superconductors Within the Strong-Coupling Limit
}

\author{
Yucel Yildirim ${ }^{1, *}$ and Wei Ku (顧威 $)^{1,2, \dagger}$ \\ ${ }^{1}$ CMPMSD, Brookhaven National Laboratory, Upton, New York 11973-5000, USA \\ ${ }^{2}$ Physics Department, State University of New York, Stony Brook, New York 11790, USA \\ (Received 4 May 2010; revised manuscript received 3 June 2011; published 15 September 2011)
}

\begin{abstract}
A generic theory of the quasiparticle superconducting gap in underdoped cuprates is derived in the strong-coupling limit, and found to describe the experimental "second gap" in absolute scale. In drastic contrast to the standard pairing gap associated with Bogoliubov quasiparticle excitations, the quasiparticle gap is shown to originate from anomalous kinetic (scattering) processes, with a size unrelated to the pairing strength. Consequently, the $k$ dependence of the gap deviates significantly from the pure $d_{x^{2}-y^{2}}$ wave of the order parameter. Our study reveals a new paradigm for the nature of the superconducting gap, and is expected to reconcile numerous apparent contradictions among existing experiments and point toward a more coherent understanding of high-temperature superconductivity.
\end{abstract}

Subject Areas: Condensed Matter Physics, Superconductivity

The recent exciting discovery of the superconducting gap in the underdoped cuprates by angle-resolved photoemission spectroscopy (ARPES) [1-12] and scanning tunneling microscopy [13-19] reveals critical clues for the puzzling high-temperature superconductivity, which has proven to be one of the most important yet challenging problems of condensed matter physics for more than two decades. Indeed, unlike the larger pseudogap, the superconducting gap closes exactly at the transition temperature $T_{c}$ [12], and shows strong correlation to the doping dependence of $T_{c}$ [3,4,20-22]. Yet, some experiments found intriguing indications that the $k$ dependence of the superconducting gap might not follow the well-established pure $d$ wave of the order parameter $[12,20]$. Obviously, a deeper understanding of the properties of the superconducting gap seen in the quasiparticle excitation spectra holds an essential key to a resolution to the long-standing problem of hightemperature superconductivity in underdoped cuprates.

Despite the intensive experimental studies, to date there have been limited attempts [20,23-27] to understand the superconducting gap, which greatly contrasts with the numerous efforts made to address the pseudogap phenomenon [28-31]. Furthermore, the existing understanding for the superconducting gap remains within the scope of the weakcoupling BCS framework, which was shown to be insufficient [32] in the underdoped regime to account for the essential phase fluctuation of the order parameter. As a result, most of the key questions remain open, including

\footnotetext{
*Present address: Physics Department, Faculty of Arts and Sciences, Doğuş University, Acıbadem-Kadıköy, 34722 Istanbul, Turkey.

†weiku@bnl.gov

Published by the American Physical Society under the terms of the Creative Commons Attribution 3.0 License. Further distribution of this work must maintain attribution to the author(s) and the published article's title, journal citation, and DOI.
}

those regarding the energy scale that controls the size of the superconducting gap, the nature of the sharp quasiparticle at the edge of the superconducting gap, and the precise $k$ dependence of the superconducting gap, among others.

Here we address the above key questions regarding the superconducting gap by deriving rigorously a generic description in the strong-coupling limit, in which the realspace pairs of holes are assumed to be tightly bound in nearest neighboring sites. With a rigorous separation of the Hilbert space into bosonic and fermionic portions that describe the bound pairs and unbound doped holes, respectively, the low-energy fermionic coupling to the Bose condensate is made explicit. The resulting size of the superconducting gap is shown to describe accurately the experimental gap in absolute scale without any free parameters. Intriguingly, the superconducting gap is found to originate from anomalous kinetic processes without direct connection to the pairing strength, which is completely different from the standard Bogoliubov quasiparticle excitation. Furthermore, the $k$ dependence of the superconducting gap deviates significantly from the pure $d_{x^{2}-y^{2}}$ wave of the underlying order parameter. This new paradigm fills a longstanding void in our knowledge on the superconducting gap in the opposite limit of the BCS theory, and is expected to reconcile existing and future experiments on the quasiparticle superconducting gap and point toward a comprehensive understanding of high- $T_{c}$ superconductivity.

Generally speaking, the low-energy physics of the strong-coupling limit should be simple and universal, since the pairing energy is too large, leaving only effective kinetics active at low energy. That is, with the help of a strong constraint resulting from the higher-energy pairing interactions, most low-energy physics should be understandable, given just the one-particle Green function. This is analogous to the absence of $U$ from the Hubbard model in the lower-energy sector. For example, in the $t$ - $J$ model it has been replaced by the strong, no-double-occupancy 
constraint. We will treat the pseudogap phase as the "normal" state in which coherent pairs are not yet formed, and extract the fully dressed kinetics by fitting the ARPES spectral functions. Then, the many-body Hilbert space of the doped holes will be split rigorously into those spanned by the paired holes (bosons) and the unpaired holes (fermions) to reveal the internal processes of the kinetics, from which the quasiparticle superconducting gap can be obtained via the fermion's coupling to the coherent pairs formed below $T_{c}$.

Figure 1 shows the tight-binding parameters $\tau_{i j}$ obtained from directly fitting [33] the available ARPES data $[11,34,35]$ in the pseudogap phase of $\mathrm{La}_{2-\delta} \mathrm{Sr}_{\delta} \mathrm{CuO}_{4}$ over a large energy $(\sim 0.7 \mathrm{eV})$ range. The corresponding dispersion is consistent with the hole spin-polaron spectrum in the $t-t^{\prime}-t^{\prime \prime}-J$ model [36]. The resulting Hamiltonian

$$
H=\sum_{i i^{\prime}} \tau_{i i^{\prime}} c_{i}^{\dagger} c_{i^{\prime}}
$$

should be properly understood as a convenient representation of the average one-particle kinetic process given by the dressed one-particle propagator under the full renormalization of the many-body interactions. It certainly does not provide the explicit information of the pairing interaction, nor the decay of the quasiparticle. As clearly shown in Fig. 1, the fully dressed band structure is doping dependent, reflecting the competition between the bare kinetic and potential energy. Note that the corresponding hopping parameters are to be distinguished from those commonly used in the Hubbard or $t-J$ models, as the large interaction-induced renormalization is fully absorbed here. Specifically, the nearest-neighbor hopping, characterized by parameter $\tau$, is almost entirely suppressed. This is understandable from the strong antiferromagnetic correlation, and is thus dropped from now on. Interestingly, the values of the second- and the third-neighbor hopping parameters, $\tau^{\prime}$ and $\tau^{\prime \prime}$, approach the same value near the $5.2 \%$ quantum critical point, where

(a)

\begin{tabular}{ccccc}
$\delta(\%)$ & 5.2 & \multicolumn{1}{c}{7} & \multicolumn{1}{c}{15} & \multicolumn{1}{c}{22} \\
\hline$\tau$ & -4.0 & -3.7 & 1.1 & 8.6 \\
$\tau^{\prime}$ & 29.8 & 30.6 & 33.5 & 35.1 \\
$\tau^{\prime \prime}$ & 29.8 & 29.0 & 25.8 & 23.9
\end{tabular}

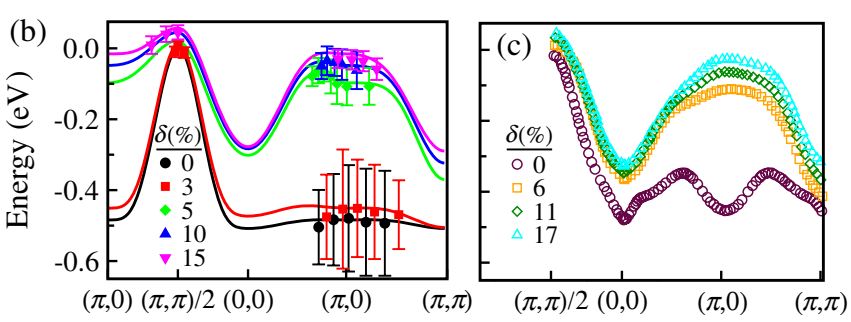

FIG. 1. Doping $(\delta)$ dependent hopping parameters (in meV) [Panel (a)] extracted from the spectral dispersion (energy vs momentum) of ARPES in the pseudogap phase of LSCO [11,34,35] (b), which resembles well the results of the exact diagonalization of the $t$ - $J$ model [36] (c). superconductivity ceases to exist even at temperature $T=0$. The equivalence of $\tau^{\prime}$ and $\tau^{\prime \prime}$ turns out to have profound influences on the behavior of the superfluid (cf., Ref [33]) and the superconducting gap as shown below.

Next, consider the strong-coupling regime where the binding of pairs is stronger than the fully renormalized kinetic energy, such that at low temperature and low energy, the paired holes cannot break apart easily. Such a regime can result from the suppression of the kinetic process mentioned above or the large potential energy associated with the antiferromagnetic correlation [37-41] and/or the formation of bipolarons [42]. (As argued above, the details of the pairing mechanism are actually not essential for the low-energy physics of the superconducting gap in this limit.) Consistent with the pure $d_{x^{2}-y^{2}}$ symmetry of the order parameter [33], in such a strong-coupling regime, the paired holes are expected to remain primarily first-neighbor to each other as real-space pairs, since occupation of a single site by two doped holes is unlikely in a lightly doped Mott insulator. Similarly, the probability of finding unpaired holes below or around $T_{c}$ is small. It is thus convenient to split the many-body Hilbert space into two components: one spanned by the tightly bound pairs of the nearest neighboring holes, $b_{i j}^{\dagger}=c_{i \uparrow}^{\dagger} c_{j l}^{\dagger}$, and the other by the unpaired holes, $f_{i \sigma}^{\dagger}$. For simplicity, a strong antiferromagnetic correlation at low energy is assumed here (which is well justified in the underdoped regime), so we will focus on the charge and pairing channels here.

Obviously, the two-site nature of the bosons introduces potential ambiguities in the representation. To ensure a unique representation, the following two conventions are

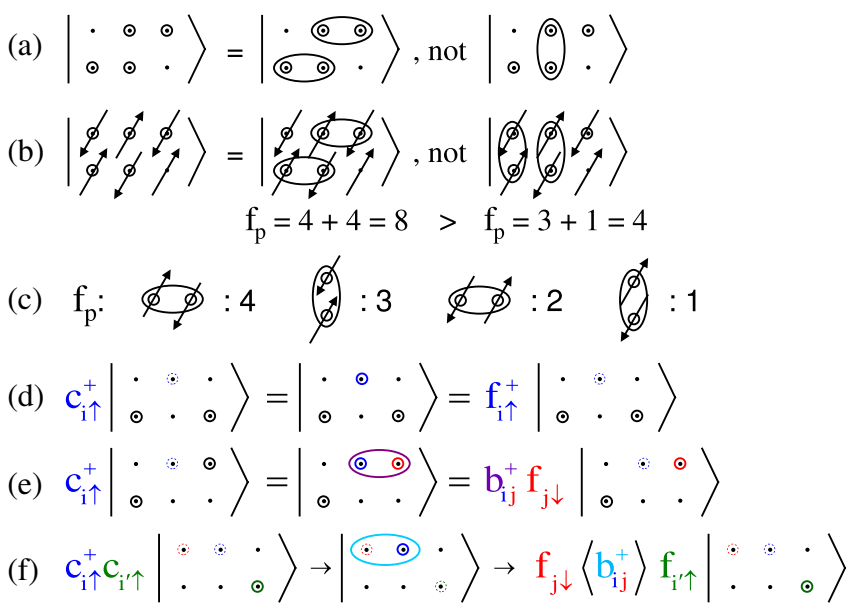

FIG. 2. Illustrations of (a) convention (A) and (b) convention (B), and the (c) definition of the preference factor $f_{p}$ for a unique representation. (d) and (e) Illustrations of two possible contributions of hole creations. (f) An example of anomalous kinetic processes, where $\left\langle b_{i j}^{\dagger}\right\rangle$ refers to the condensate component only. Dots denote the fermionic sites and the dotted circles indicate sites to be filled by doped holes. Solid circles denote the doped holes and ellipses mark the hole pairs. 
introduced: (A) whenever possible, the bosonic representation is employed prior to the fermionic one [cf., Fig. 2(a)], and (B) if multiple representations still exist, the one that maximizes the total "preference factor" is employed [cf., Fig. 2(b)], where the preference factor is assigned to each pair according to the convention defined in Fig. 2(c). Convention (B) is chosen to allow a one-on-one mapping in the representation for simpler counting. An alternative choice would be to take the symmetric superposition of all the equivalent representations, normalized to ensure correct counting.

In the new representation, the original creation operator of a doped hole can be partitioned rigorously into

$$
\begin{aligned}
& c_{i \uparrow}^{\dagger}=f_{i \uparrow}^{\dagger}+\sum_{j \in N N(i)}^{\prime} b_{i j}^{\dagger} f_{j \downarrow}+\left[\sum b^{\dagger} b^{\dagger} b f+\ldots\right], \\
& c_{j \downarrow}^{\dagger}=f_{j \downarrow}^{\dagger}-\sum_{i \in N N(j)}^{\prime} b_{i j}^{\dagger} f_{i \uparrow}+\left[\sum b^{\dagger} b^{\dagger} b f+\ldots\right],
\end{aligned}
$$

where $\Sigma^{\prime}$ denotes the "sum over only the first nonzero term" according to the convention defined above. Figures 2(d) and 2(e) illustrates the meaning of this nonstandard expression. When creating an additional hole, one can either (first term) create an unpaired hole if and only if no other unpaired hole is next to it [Fig. 2(d)], or (second term) meet an unpaired hole and form a new pair [Fig. 2(e)]. It is important to emphasize the mutual exclusion of the terms in Eq. (2) according to the above conventions [43]. Owing to the low density of the doped holes, the higherorder terms can be dropped safely. For a cleaner presentation, the spin indices and the associated sign changes will be suppressed from now on, and the addition of Hermitian conjugate will be implied.

Applying Eq. (2) to Eq. (1) and dropping the redundant $f_{j} f_{j}^{\dagger}$ in $b_{i j}^{\dagger} f_{j} f_{j}^{\dagger} b_{i^{\prime} j}$, the effective one-particle kinetics then becomes

$$
\begin{aligned}
H= & H^{b}+H^{f}+H^{b f} \\
= & \left(\sum_{i i^{\prime} j} \tau_{i i^{\prime}} b_{i j}^{\dagger} b_{i^{\prime} j}\right)+\left(\sum_{i i^{\prime}} \tau_{i i^{\prime}} f_{i}^{\dagger} f_{i^{\prime}}\right) \\
& +\left(\sum_{i i^{\prime}} \sum_{j}^{\prime} \tau_{i i^{\prime}} b_{i j}^{\dagger} f_{j} f_{i^{\prime}}+\sum_{i i^{\prime}} \sum_{j j^{\prime} \neq j}^{\prime} \tau_{i i^{\prime}} b_{i j}^{\dagger} f_{j} f_{j^{\prime}}^{\dagger} b_{i^{\prime} j^{\prime}}\right),
\end{aligned}
$$

where $H^{b}$ describes the pivoting motion of the pairs that results in the local $d$-wave symmetry [33], $H^{f}$ gives the motion of unpaired holes, and $H^{b f}$ describes the coupling between the bosons and the fermions.

It is important to distinguish Eq. (3) from an ad hoc fermion-boson model, as Eq. (3) is derived rigorously from Eq. (1) and (2), with $b^{\dagger}$ and $f^{\dagger}$ originating from projections of the same $c^{\dagger}$ operator. Consequently, the fermion and boson operators do not commute in general, and are forbidden to occupy the same site. We stress that Eq. (3) is only meant to reveal the internal kinetic processes embedded in the one-particle propagator represented by Eq. (1). For example, $H^{b f}$ gives the irreducible scattering kernel between fermion and boson propagators.

Obviously, at $T>T_{c}$ the one-body propagator $G(t, 0) \equiv\left\langle c(t) c^{\dagger}(0)\right\rangle=\left\langle f(t) f^{\dagger}(0)\right\rangle+\left\langle f^{\dagger}(t) b(t) f^{\dagger}(0)\right\rangle+$ $\left\langle f(t) b^{\dagger}(0) f(0)\right\rangle+\left\langle f^{\dagger}(t) b(t) b^{\dagger}(0) f(0)\right\rangle$ recovers the original experimental dispersion by construction. (Here \langle\rangle denotes the time-ordered ensemble average.) At low energy, the last three terms of $G$ are insignificant because of (1) the large pairing/depairing energy they need and (2) the insufficient supply of unpaired holes. This leaves only the first term in $G$ essential to the low-energy physics of the quasiparticle, corresponding to the injection of an unpaired hole followed by the removal of the same at some other time.

At $T<T_{c}$, the formation of coherent pairs introduces additional effects on the low-energy fermionic Hilbert space through terms such as the third term in Eq. (3). Given a zero momentum $(q=0)$ condensate with a $d_{x^{2}-y^{2}}$ phase, as the experimentally observed ones, the corresponding $\left\langle b_{q=0}^{\dagger}\right\rangle$ becomes macroscopic. In turn, additional anomalous kinetic processes [Fig. 2(f) for an example] are enabled, whose Fourier transform gives $\sqrt{n_{0}} \sum_{k} t_{k}^{b f} f_{k} f_{-k}+$ c.c. (upon averaging $\Sigma^{\prime} \rightarrow \frac{1}{4} \Sigma$ ). Here $n_{0}=\left.\left\langle b_{q}^{\dagger} b_{q}\right\rangle\right|_{q=0}$ denotes the number of pairs per $\mathrm{Cu}$ atom in the condensate, and may be practically approximated by the measured superfluid density $n_{s}$ (in number/Cu). (One should keep in mind that $n_{0}$ is, in general, smaller than $n_{s}$, especially at a higher density, such as in the overdoped regime.) The $k$-dependent fermioncondensate coupling,

$$
\begin{aligned}
t_{k}^{b f}= & \frac{1}{2}\left[\cos \left(k_{x}\right)-\cos \left(k_{y}\right)\right] \\
& \times\left\{4 \tau^{\prime} \cos \left(k_{x}\right) \cos \left(k_{y}\right)+2 \tau^{\prime \prime}\left[\cos \left(2 k_{x}\right)+\cos \left(2 k_{y}\right)\right]\right\},
\end{aligned}
$$

has an easily recognized structure: the first part originates from the $x^{2}-y^{2}$ structure of the order parameter, while the second originates from the Fourier transform of the hopping processes of various ranges. (Notation $t$ is used here, instead of $\tau$, to emphasize that this coupling is only to the condensate component of the bosonic space.) Finally, the standard Bogoliubov transformation leads to our main result on the low-energy quasiparticle gap:

$$
\Delta_{k}(T)=t_{k}^{b f} \sqrt{n_{0}(T)} .
$$

Several striking features emerge from this rigorous and generic derivation of the quasiparticle superconducting gap in the strong-coupling regime. First, in drastic contrast to the weak-coupling BCS theory, the superconducting gap is controlled directly by the strength of the anomalous kinetic process, $t_{k}^{b f}$, not the pairing strength. To verify this unconventional conclusion, in Fig. 3(a) we compare our result directly with the ARPES [11] experimental superconducting gap of $\mathrm{La}_{2-\delta} \mathrm{Sr}_{\delta} \mathrm{CuO}_{4}$ (LSCO) at $T=7.6 \mathrm{~K} \sim 0.54 T_{c}$ $\left(T_{c}=14 \mathrm{~K}\right)$ for $\delta \sim 7 \%$. The theoretical $n_{0}(T)$ is estimated 
from $\theta \gamma \frac{\delta}{2}$, where the thermal reduction factor $\theta=$ $n_{0}\left(0.54 T_{c}\right) / n_{0}(0) \sim n_{s}\left(0.54 T_{c}\right) / n_{s}(0) \sim 0.72$ is taken directly from the penetration depth measurement of YBCO at the same doping level [44]. (We are unaware of similar data for LSCO.) The quantum reduction factor $\gamma=$ $n_{0}(0) / n_{\text {total }}$ accounts for the loss due to the population of the finite momentum state in interacting bosonic systems and is only suppressed noticeably from 1 near the $5.2 \%$ quantum critical point. Here $\gamma(\delta=7 \%) \sim 0.85$ is taken from Ref. [33].

Clearly, Fig. 3 shows an excellent agreement with the experimental superconducting gap in absolute scale. Considering that not a single free parameter is needed in the theory, this degree of agreement is truly remarkable. Evidently, the superconducting gap measured by ARPES and scanning tunneling microscopy (and other spectroscopies) reflects the strength of the effective kinetics of the doped holes, instead of the pairing strength, a feature unimaginable from the weak-coupling BCS theory.

The $(\delta, k)$-dependence of the superconducting gap holds another surprise in our result. As already hinted near the end of the experimental data set $\left(\phi \sim 30^{\circ}\right)$ in Fig. 3(a), the $k$ dependence of the theoretical superconducting gap deviates dramatically from that of the pure $d_{x^{2}-y^{2}}$ symmetry of the underlying order parameter (cf., the fitted line in green). Unexpectedly, the size of the superconducting gap actually reduces near $(\pi, 0)$ instead of reaching its maximum. A clearer picture is demonstrated in Fig. 3(b) for three doping levels, $\delta=22 \%$ (overdoped), $15 \%$ (optimally doped), and $5.2 \%$ (extremely underdoped). For a
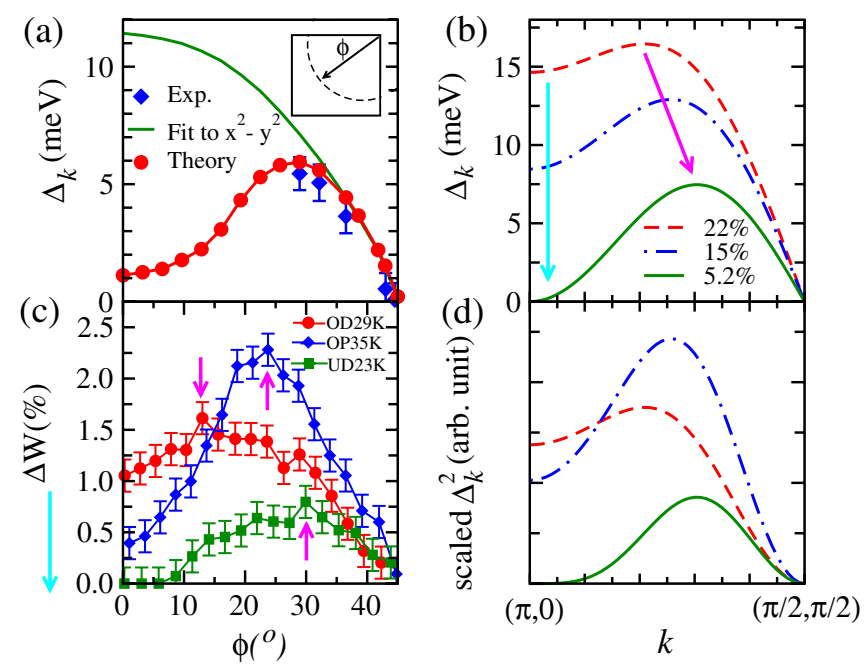

FIG. 3. (a) Momentum dependence of the superconducting gap for $7 \%$ doped LSCO at $7.6 \mathrm{~K}$. Momentum is given in $\phi$, see inset. (b) Theoretical $k$ dependence of the superconducting gap from overdoped $(\mathrm{OD} / 22 \%)$, optimally doped (OP/15\%), and underdoped (UD/5.2\%) systems. (c) ARPES normalized weight transfer $(\Delta W)$ in Bi2201 [12]. (d) Scaled theoretical $\Delta^{2}$ as a simulation of $\Delta W$ outside the Fermi arc. Blue and pink arrows indicate the trends upon reduced doping. clearer illustration of the trend, $n_{0}$ is simply set to $\delta / 2$. (Thus, an exaggeration of the overall scale of the superconducting gap for the $22 \%$ case is to be understood.)

Two apparent trends can be easily identified from Fig. 3(b). First, the relative size of the superconducting gap at $(\pi, 0)$ decreases with the reduction in the doping level. In fact, Eq. (4) indicates that near the quantum critical point at $\delta \sim 5.2 \%$, where $\tau^{\prime}$ and $\tau^{\prime \prime}$ approach the same value [cf. Fig. 1, panel (a)], the size of the superconducting gap reduces to exactly zero at $(\pi, 0)$. Second, the $k$ point at which the superconducting gap reaches its maximum on the Fermi surface shifts toward $(\pi / 2, \pi / 2)$ as $\delta$ decreases, in a manner similar to the end of the Fermi arc.

These predicted trends need to be checked by future experiments, as it has proven challenging with the current ARPES resolution to quantify $\Delta$ reliably outside the Fermi arc where no sharp peak is preset to allow a clean identification of the gap. Particularly, deep within the pseudogap, where the normal-state spectral function $A(\omega) \propto \omega^{2}$, an opening of a small superconducting gap would not induce a clear spectral enhancement right above $\Delta$, but only gives a slight weight transfer from inside the pseudogap to higher energy (see the Appendix). An available alternative at the moment is thus to compare the spectral weight transfer $\Delta W$ [12] from $T>T_{c}$ to $T \ll T_{c}$. As elaborated in the Appendix, $\Delta W$ is sensitive to the size of $\Delta$ : inside the Fermi arc, where the spectral function is large at the chemical potential, $\Delta W \propto \Delta$, while outside the Fermi arc $\Delta W \propto \Delta^{2}$. Figure 3(d) shows $\Delta^{2}$ with $\Delta(\delta=22 \%)$ scaled by $75 \%$ to account for the above mentioned overestimation of $n_{0}$ by $\delta / 2$ to give a more realistic visualization. Clearly, these two theoretical trends remain robust and should be present in the experimental spectral weight transfer.

Encouragingly, both trends appear to be observed clearly in the recent ARPES analysis [12] of $\Delta W$ in $(\mathrm{Bi}, \mathrm{Pb})_{2} \times$ $(\mathrm{Sr}, \mathrm{La})_{2} \mathrm{CuO}_{6+\delta}$, which is shown in Fig. 3(c). Other than an overall scale introduced by the chosen reference for experimental normalization, our theoretical results resemble very much the experimental $(\delta, k)$ dependence. In particular, they capture both predicted trends that are entirely unexpected from a pure $d_{x^{2}-y^{2}}$ symmetry of the underlying order parameter, another feature inconceivable from the weak-coupling BCS theory.

These unconventional features in our resulting superconducting gap reflect a new scenario of the quasiparticle superconducting gap in the strong-coupling limit, as revealed in our derivation. Indeed, the smaller superconducting gap observed inside the pseudogap has a novel character entirely different from the well-understood Bogoliubov quasiparticle excitation in weak-coupling theories [cf., Figs. 4(a) and 4(b)] that breaks apart a particle from the pair with an energy scale of the binding energy. In Eq. (3) the coupling $b_{i j}^{\dagger} f_{j} f_{i^{\prime}}=\left(g_{j} b_{i j}\right)^{\dagger} f_{i^{\prime}}$ allows an additional low-energy anomalous kinetic process [Figs. 2(f), 4(c), and $4(\mathrm{~d})]$ of an unpaired hole $f^{\dagger}$ hopping and morphing 
(a)

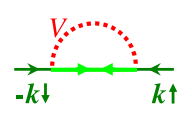

(c)

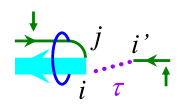

(d)
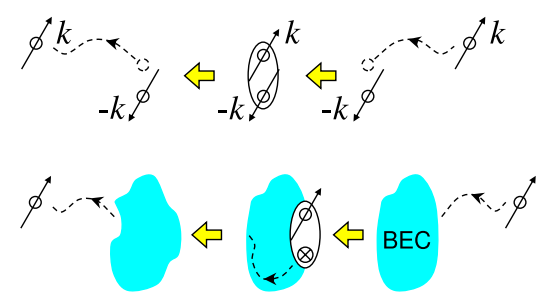

FIG. 4. (a) An example of anomalous coupling via pairing interaction $V$ in the BCS theory, with green lines denoting $G$ of momentum $k$. (b) Illustration of the Bogoliubov quasiparticle. (c) The anomalous kinetic coupling in this work, with a thick blue line denoting the bosonic propagator of the condensate and a thin blue ring emphasizing the antihole's confinement to the condensate. (d) Illustration of the low-energy quasiparticle below the pairing energy scale.

into an antihole $g^{\dagger} \equiv f$ inside the Bose-Einstein condensate (BEC), and its Hermitian conjugate allows the reverse process of an antihole hopping into a hole outside the BEC. Since the creation of the composite object $\left(g_{j} b_{i j}\right)^{\dagger}$ does not involve the energy of the pairing strength (unlike the creation of a single $b^{\dagger}$ ), the entire effective scattering process [Figs. 4(c) and 4(d)] only reflects the energy scale of the kinetics $\tau_{i i^{\prime}}$. In other words, the superconducting gap here is not a pairing gap, but a scattering gap, whose size reflects the energy scale required for the low-energy carriers to overcome the coherent scattering against the condensate.

An important implication of the above new scenario is that the low-energy quasiparticles, especially those around the superconducting gap, are distinct from the holes that form the pairs. Thus, they cannot participate in the decay of pairs via the standard depairing process [45] advocated previously in order to explain the near-linear reduction of the superfluid density against the temperature [44]. This leaves thermal phase fluctuation [32] or thermal depletion of the BEC [33] as the only known explanations. Furthermore, even though both $T_{c}$ [33] and the superconducting gap are unrelated to the pairing strength, they are correlated via $n_{s}(0): T_{c} \propto n_{s}(0)[32,33]$ and $\Delta \propto \sqrt{n_{0}(0)} \sim$ $\sqrt{n_{s}(0)}$, again defying $\Delta / T_{c} \sim 1.8$ from the BCS theory.

Obviously, the novel nature of the superconducting gap strongly suggests revisiting the existing theoretical considerations and experimental interpretations of the superconducting gap. In particular, the different $k$ dependence of the superconducting gap and the order parameter raises an alarming flag on the common practice of equating the superconducting gap to the order parameter. Similarly, the kinetic origin of the superconducting gap renders inapplicable the practice of regarding the power-law decay of low-temperature superfluid density as evidence of nodes. Finally, the above generic considerations can be applied to the analysis of other strong interaction theories [36,46] to provide further physical insights unapparent from their numerical solutions.
In conclusion, we develop a generic theory of the superconducting gap in the strong-coupling limit suitable for underdoped cuprates. The resulting superconducting gap is shown to be a scattering gap, fundamentally distinct from the pairing gap associated with Bogoliubov quasiparticle excitations in the standard theories. Not only is its size controlled directly by the anomalous kinetic process instead of the pairing strength, but its $(\delta, k)$ dependence also deviates significantly from the $d_{x^{2}-y^{2}}$ wave of the underlying order parameter. The new theory fills the longstanding void in our knowledge on the superconducting gap in the opposite limit of the BCS theory and reveals the essential nature of the high- $T_{c}$ superconductivity.

We thank A. Kaminski and T. Kondo for providing the experimental data in Fig. 3(c), Chia-Hui Lin for discussions on the weakening of signals of $\Delta$ in the pseudogap phase, and H. Yang and J. Rameau for helpful discussions on the experimental observations of ARPES. This work was supported by the U.S. Department of Energy, Office of Basic Energy Science, under Contract No. DE-AC0298CH10886.

\section{APPENDIX: ASSIGNING $\Delta$ WITH MISLEADING WIDE RANGE OF SPECTRAL WEIGHT LOSS IN THE PSEUDOGAP}

Here we reiterate the points from Ref. [47] on the misleading assignment of $\Delta$ inside a large pseudogap, and indicate why the approximate spectral weight transfer $\Delta W$ serves as an alternative measure of $\Delta$.

From Eqs. (3)-(5) above, the one-particle spectral function in the normal state $A(k, \omega)$ will be altered to

$$
A(k, \Omega)=A(k, \omega) \frac{d \omega}{d \Omega}=A(k, \omega) \frac{\Omega}{\omega},
$$

below $T_{c}$, with $\omega^{2}=\Omega^{2}-\Delta^{2}$. Here $A(k, \Omega)=0$ for $|\Omega|<\Delta$ is implied, and $\Omega$ is the energy of the photo hole. In the simple case of $A(k, \omega)=\delta\left(\omega-\epsilon_{k}\right)$ or $A(k, \omega)=c$ near the chemical potential, one expects to observe a clear signature of $\Delta$ via the enhancement of the quasiparticle spectrum at $|\Omega| \sim \Delta$ since $A(k, \Omega)=$ $\delta\left(\Omega-\sqrt{\epsilon_{k}^{2}+\Delta^{2}}\right)$ and $c \Omega / \sqrt{\Omega^{2}-\Delta^{2}}$, respectively, which is shown in Figs. 5(a) and 5(b). However, the signature of $\Delta$ reduces significantly in the higher order polynomial contribution to $A$. For example, the first order contribution $A(k, \omega)=c_{k} \omega$ leads to $A(k, \Omega)=c_{k} \Omega$, which is shown in Fig. 5(c). Other than the removal of the spectral weight from within the gap, one sees no enhancement of the weight near the edge of the gap.

Even more dramatically, for $A(k, \omega)=c_{k} \omega^{2}$ to be relevant for the low-energy part within the pseudogap at lowtemperature, one finds that $A(k, \Omega)=c_{k} \Omega \sqrt{\Omega^{2}-\Delta^{2}}$ has reduced spectral weight for a deceivingly wide range of energy much larger than $\Delta$ [cf., Fig. 5(d)]. There is no 


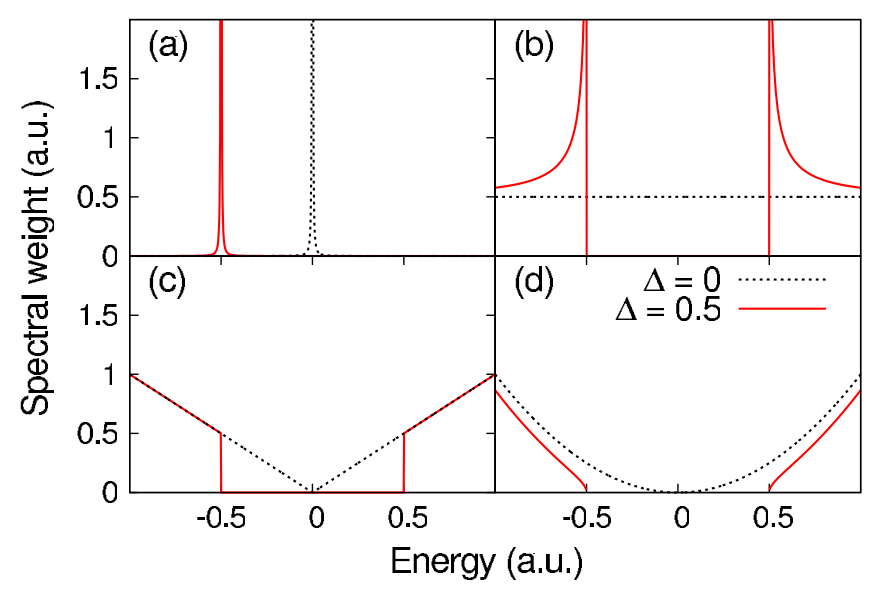

FIG. 5. Spectral functions in the superconducting phase (solid lines; $\Delta=0.5$ ) derived from that of the normal state (dotted lines; $\Delta=0$ ): (a) $\delta$ function, (b) constant, (c) linear, and (d) quadratic.

simple peaklike signal for identifying $\Delta$ in the spectral function. Furthermore, from Fig. 6, where the situation with a pseudogap is simulated, one sees that the common practice of using leading edge or weight crossing to identify $\Delta$ encounters significant difficulty as well. The original weight within $\Delta$ is transferred to a very high energy, basically, to the energy where the normal state $A(k, \omega)$ turns into sublinear power in $\omega$. Specifically, in the pseudogap case, the weight enhancement occurs at $\Omega$ around the scale of the pseudogap, rather than at $\Delta$. This explains why most of the standard ARPES analyses are often misled into concluding that $\Delta$ is very close to the pseudogap size in this region, even though the weight transfer is negligibly small.

Nonetheless, Eqs. (3)-(5) still lead to a spectral weight transfer (when $\Delta$ is much less than the pseudogap)

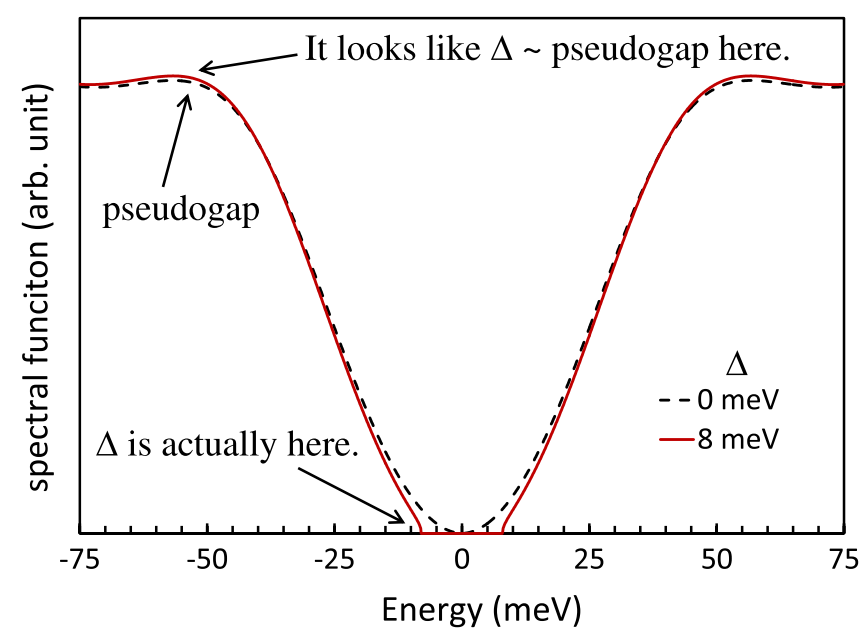

FIG. 6. Spectral function of the superconducting phase (solid line) derived from that of the normal state (dashed line) in the presence of a pseudogap.
$\Delta W \sim \int_{0}^{\Delta_{p}} c_{k} \omega^{2} d \omega-\int_{\Delta_{k}}^{\Delta_{p}} c_{k} \omega \sqrt{\omega^{2}-\Delta_{k}^{2}} d \omega \rightarrow c_{k} \Delta_{p} \Delta_{k}^{2} / 2$

sensitive to $\Delta_{k}$, with $\Delta_{p}$ denoting the energy where the weight crossing takes place, of the order of the pseudogap. Thus, $\Delta W$ can serve as an approximate measure of $\Delta_{k}$ after proper normalization and scaling.

In short, in the presence of a large pseudogap, there is no simple feature in the spectral function that can be used to identify the size of $\Delta$; there is no strong peaklike structure, nor weight enhancement near $\Omega \sim \Delta$. The most apparent way to estimate $\Delta$ is through a normalized spectral weight transfer [12]. Ultimately, a true quantification of $\Delta$ would require a careful spectral analysis that takes into account the change of line shape via Eq. (A1).

[1] K. M. Shen et al., Nodal Quasiparticles and Antinodal Charge Ordering in $\mathrm{Ca}_{2-x} \mathrm{Na}_{x} \mathrm{CuO}_{2} \mathrm{Cl}_{2}$, Science 307, 901 (2005).

[2] A. Kanigel et al., Evolution of the Pseudogap from Fermi Arcs to the Nodal Liquid, Nature Phys. 2, 447 (2006).

[3] K. Tanaka et al., Distinct Fermi-Momentum-Dependent Energy Gaps in Deeply Underdoped Bi2212, Science 314, 1910 (2006).

[4] M. Le Tacon, A. Sacuto, A. Georges, G. Kotliar, Y. Gallais, D. Colson, and A. Forget, Two Energy Scales and Two Distinct Quasiparticle Dynamics in the Superconducting State of Underdoped Cuprates, Nature Phys. 2, 537 (2006).

[5] B. Goss Levi, New Experiments Fuel Debate Over the Nature of High- $T_{c}$ Superconductors, Phys. Today 60, 17 (2007).

[6] T. Kondo, T. Takeuchi, A. Kaminski, S. Tsuda, and S. Shin, Evidence for Two Energy Scales in the Superconducting State of Optimally Doped, $(\mathrm{Bi}, \mathrm{Pb})_{2}(\mathrm{Sr}, \mathrm{La})_{2} \mathrm{CuO}_{6+\delta}$, Phys. Rev. Lett. 98, 267004 (2007).

[7] W. S. Lee, I. M. Vishik, K. Tanaka, D. H. Lu, T. Sasagawa, N. Nagaosa, T.P. Devereaux, Z. Hussain, and Z.-X. Shen, Abrupt Onset of a Second Energy Gap at the Superconducting Transition of Underdoped Bi2212, Nature (London) 450, 81 (2007).

[8] K. Terashima, H. Matsui, T. Sato, T. Takahashi, M. Kofu, and K. Hirota, Anomalous Momentum Dependence of the Superconducting Coherence Peak and Its Relation to the Pseudogap of $\mathrm{La}_{1.85} \mathrm{Sr}_{0.15} \mathrm{CuO}_{4}$, Phys. Rev. Lett. 99, 017003 (2007).

[9] M. Hashimoto, T. Yoshida, K. Tanaka, A. Fujimori, M. Okusawa, S. Wakimoto, K. Yamada, T. Kakeshita, H. Eisaki, and S. Uchida, Distinct Doping Dependences of the Pseudogap and Superconducting Gap of $\mathrm{La}_{2-x} \mathrm{Sr}_{x} \mathrm{CuO}_{4}$ Cuprate Superconductors, Phys. Rev. B 75, 140503(R) (2007).

[10] S Hüfner, M A Hossain, A Damascelli, and G A Sawatzky, Two Gaps Make a High-Temperature Superconductor?, Rep. Prog. Phys. 71, 062501 (2008). 
[11] T. Yoshida et al., Universal versus Material-Dependent Two-Gap Behaviors of the High- $T_{c}$ Cuprate Superconductors: Angle-Resolved Photoemission Study of $\mathrm{La}_{2-x} \mathrm{Sr}_{x} \mathrm{CuO}_{4}$, Phys. Rev. Lett. 103, 037004 (2009).

[12] T. Kondo, R. Khasanov, T. Takeuchi, J. Schmalian, and A. Kaminski, Competition Between the Pseudogap and Superconductivity in the High- $T_{c}$ Copper Oxides, Nature (London) 457, 296 (2009).

[13] J.E. Hoffman, K. McElroy, D.-H. Lee, K. M. Lang, H. Eisaki, S. Uchida, and J. C. Davis, Imaging Quasiparticle Interference in $\mathrm{Bi}_{2} \mathrm{Sr}_{2} \mathrm{CaCu}_{2} \mathrm{O}_{8+\delta}$, Science 297, 1148 (2002).

[14] K. McElroy, R. W. Simmonds, J. E. Hoffman, D.-H. Lee, J. Orenstein, H. Eisaki, S. Uchida, and J.C. Davis, Relating Atomic-Scale Electronic Phenomena to Wave-Like Quasiparticle States in Superconducting $\mathrm{Bi}_{2} \mathrm{Sr}_{2} \mathrm{CaCu}_{2} \mathrm{O}_{8+\delta}$, Nature (London) 422, 592 (2003).

[15] T. Hanaguri, Y. Kohsaka, J.C. Davis, C. Lupien, I. Yamada, M. Azuma, M. Takano, K. Ohishi, M. Ono, and H. Takagi, Quasiparticle Interference and Duperconducting Gap in $\mathrm{Ca}_{2-x} \mathrm{Na}_{x} \mathrm{CuO}_{2} \mathrm{Cl}_{2}$, Nature Phys. 3, 865 (2007).

[16] K. K. Gomes , A. N. Pasupathy, A. Pushp, S. Ono, Y. Ando, and A. Yazdani, Visualizing Pair Formation on the Atomic Scale in the High- $T_{c}$ Superconductor $\mathrm{Bi}_{2} \mathrm{Sr}_{2} \mathrm{CaCu}_{2} \mathrm{O}_{8+\delta}$, Nature (London) 447, 569 (2007).

[17] A. N. Pasupathy, A. Pushp, K. K. Gomes, C. V. Parker, J. Wen, Z. Xu, G. Gu, S. Ono, Y. Ando, and A. Yazdani, Electronic Origin of the Inhomogeneous Pairing Interaction in the High- $T_{c}$ Superconductor $\mathrm{Bi}_{2} \mathrm{Sr}_{2} \mathrm{CaCu}_{2} \mathrm{O}_{8+\delta}$, Science 320, 196 (2008).

[18] Y. Kohsaka et al., How Cooper Pairs Vanish Approaching the Mott Insulator in $\mathrm{Bi}_{2} \mathrm{Sr}_{2} \mathrm{CaCu}_{2} \mathrm{O}_{8+\delta}$, Nature (London) 454, 1072 (2008).

[19] M. C. Boyer, W. D. Wise, K. Chatterjee, M. Yi, T. Kondo, T. Takeuchi, H. Ikuta, and E.W. Hudson, Imaging the Two Gaps of the High-Temperature Superconductor $\mathrm{Bi}_{2} \mathrm{Sr}_{2} \mathrm{CuO}_{6+x}$, Nature Phys. 3, 802 (2007).

[20] J. Mesot et al., Superconducting Gap Anisotropy and Quasiparticle Interactions: A Doping Dependent Photoemission Study, Phys. Rev. Lett. 83, 840 (1999).

[21] G. Deutscher, Coherence and Sngle-Particle Excitations in the High-Temperature Superconductors, Nature (London) 397, 410 (1999).

[22] M. Opel et al., Carrier Relaxation, Pseudogap, and Superconducting Gap in High- $T_{c}$ Cuprates: A Raman Scattering Study, Phys. Rev. B 61, 9752 (2000).

[23] J. Quintanilla and B.L. Györffy, On the Nature of the Superconducting Gap in the Cuprates, J. Phys. Condens. Matter 14, 6591 (2002).

[24] T. Das, R. S. Markiewicz, and A. Bansil, Competing Order Scenario of Two-Gap Behavior in Hole-Doped Cuprates, Phys. Rev. B 77, 134516 (2008).

[25] M. Gomes and A. Ghosh, Asymmetric Superconducting Gap and DDW State in High-T $T_{c}$ Cuprates, Eur. Phys. J. B 66, 461 (2008).

[26] C.-C. Chien, Y.He, Q. Chen, and K. Levin, Two-Energy-Gap Preformed-Pair Scenario for Cuprate Superconductors: Implications for Angle-Resolved Photoemission Spectroscopy, Phys. Rev. B 79, 214527 (2009).
[27] Z. Tešanović, $d$-wave Duality and its Reflections in HighTemperature Superconductors, Nature Phys. 4, 408 (2008).

[28] H. Ding, T. Yokoya, J. C. Campuzano, T. Takahashi, M. Randeria, M. R. Norman, T. Mochiku, K. Kadowaki, and J. Giapintzakis, Spectroscopic Evidence for a Pseudogap in the Normal State of Underdoped High-T Superconductors, Nature (London) 382, 51 (1996).

[29] T. Timusk and B. Statt, The Pseudogap in HighTemperature Superconductors: An Experimental Survey, Rep. Prog. Phys. 62, 61 (1999).

[30] A. Damascelli, Z. Hussain, and Z.-X. Shen, AngleResolved Photoemission Studies of the Cuprate Superconductors, Rev. Mod. Phys. 75, 473 (2003).

[31] Ø. Fischer, M. Kugler, I. Maggio-Aprile, Christophe Berthod, and Christoph Renner, Scanning Tunneling Spectroscopy of High-Temperature Superconductors, Rev. Mod. Phys. 79, 353 (2007).

[32] V.J. Emery and S. A. Kivelson, Importance of Phase Fluctuations in Superconductors with Small Superfluid Density, Nature (London) 374, 434 (1995).

[33] Y. Yildirim and W. Ku, arXiv:1005.0030.

[34] A. Ino, C. Kim, M. Nakamura, T. Yoshida, T. Mizokawa, Z.-X. Shen, A. Fujimori, T. Kakeshita, H. Eisaki, and S. Uchida, Electronic Structure of $\mathrm{La}_{2-x} \mathrm{Sr}_{x} \mathrm{CuO}_{4}$ in the Vicinity of the Superconductor-Insulator Transition, Phys. Rev. B 62, 4137 (2000); Doping-Dependent Evolution of the Electronic Structure of $\mathrm{La}_{2-x} \mathrm{Sr}_{x} \mathrm{CuO}_{4}$ in the Superconducting and Metallic Phases, 65, 094504 (2002).

[35] T. Yoshida et al., Systematic Doping Evolution of the Underlying Fermi Surface of $\mathrm{La}_{2-x} \mathrm{Sr}_{x} \mathrm{CuO}_{4}$, Phys. Rev. B 74, 224510 (2006).

[36] W.-G. Yin, C.-D. Gong, and P. W. Leung, Origin of the Extended Van Hove Region in Cuprate Superconductors, Phys. Rev. Lett. 81, 2534 (1998).

[37] Ch. Niedermayer, C. Bernhard, T. Blasius, A. Golnik, A. Moodenbaugh, and J.I. Budnick, Common Phase Diagram for Antiferromagnetism in $\mathrm{La}_{2-x} \mathrm{Sr}_{x} \mathrm{CuO}_{4}$ and $\mathrm{Y}_{1-x} \mathrm{Ca}_{x} \mathrm{Ba}_{2} \mathrm{Cu}_{3} \mathrm{O}_{6}$ as Seen by Muon Spin Rotation, Phys. Rev. Lett. 80, 3843 (1998).

[38] E. Dagotto, A. Nazarenko, and A. Moreo, Antiferromagnetic and Van Hove Scenarios for the Cuprates: Taking the Best of Both Worlds, Phys. Rev. Lett. 74, 310 (1995).

[39] P. Wróbel, R. Eder, and R. Micnas, Kinetic Energy Driven Superconductivity and the Pseudogap Phase in Weakly Doped Antiferromagnets, J. Phys. Condens. Matter 15, 2755 (2003).

[40] A. Mihlin and A. Auerbach, Temperature Dependence of the Order Parameter of Cuprate Superconductors, Phys. Rev. B 80, 134521 (2009).

[41] E. Altman and A. Auerbach, Plaquette Boson-Fermion Model of Cuprates, Phys. Rev. B 65, 104508 (2002).

[42] A. Alexandrov, Theory of Superconductivity from Weak to Strong Coupling, (IoP Publishing, Bristol, 2003), p. 320, ISBN 0750308362.

[43] It might be convenient to keep in mind the projection nature of $f_{i}^{\dagger} \sim Q_{i}^{b} c_{i}^{\dagger} P_{i}^{f}$. Here $P_{i}^{f} \equiv \prod_{j \in N N(i)}\left(1-n_{j}^{f}\right)$ and $Q_{i}^{b} \equiv \prod_{j \in N N(i)}\left(1-n_{i j}^{b}\right)$ are projection operators that ensure a unique representation, with $n_{j}^{f}=f_{j}^{\dagger} f_{j}$ and 
$n_{i j}^{b}=b_{i j}^{\dagger} b_{i j}$ referring to the fermion and boson number density, respectively.

[44] W. N. Hardy, D. A. Bonn, D. C. Morgan, R. Liang, and K. Zhang, Precision Measurements of the Temperature Dependence of $\lambda$ in $\mathrm{YBa}_{2} \mathrm{Cu}_{3} \mathrm{O}_{6.95}$ : Strong Evidence for Nodes in the Gap Function, Phys. Rev. Lett. 70, 3999 (1993); D. M. Broun, W. A. Huttema, P. J. Turner, S. Özcan, B. Morgan, R. Liang, W. N. Hardy, and D. A. Bonn, Superfluid Density in a Highly Underdoped $\mathrm{YBa}_{2} \mathrm{Cu}_{3} \mathrm{O}_{6+y}$ Superconductor, Phys. Rev. Lett. 99, 237003 (2007).
[45] P. A. Lee and X.-G. Wen, Unusual Superconducting State of Underdoped Cuprates, Phys. Rev. Lett. 78, 4111 (1997).

[46] M. Civelli, M. Capone, A. Georges, K. Haule, O. Parcollet, T.D. Stanescu, and G. Kotliar, NodalAntinodal Dichotomy and the Two Gaps of a Superconducting Doped Mott Insulator, Phys. Rev. Lett. 100, 046402 (2008).

[47] C.-H. Lin and W. Ku, Is Superconducting Gap Near $(\pi, 0)$ in Underdoped Cuprates as Large as the Pseudogap According to the ARPES Measurement? (to be published). 